\title{
Current-mode deep level transient spectroscopy of a semiconductor nanowire field- effect transistor
}

Ivan Isakov, Marion J. L. Sourribes, and Paul A. Warburton

Citation: Journal of Applied Physics 122, 094305 (2017); doi: 10.1063/1.5000370

View online: $\mathrm{http}: / / d x . d o i . o r g / 10.1063 / 1.5000370$

View Table of Contents: http://aip.scitation.org/toc/jap/122/9

Published by the American Institute of Physics

\section{Articles you may be interested in}

Growth and characterization of $\beta-\mathrm{Ga}_{2} \mathrm{O}_{3}$ thin films by molecular beam epitaxy for deep-UV photodetectors Journal of Applied Physics 122, 095302 (2017); 10.1063/1.4985855

Oxygen- and photoresist-related interface states of 4H-SiC Schottky diode observed by deep-level transient spectroscopy

Journal of Applied Physics 122, 094504 (2017); 10.1063/1.4989912

Decoupling recombination mechanisms and trap state localization in direct bandgap semiconductors using photoluminescence decay

Journal of Applied Physics 122, 095705 (2017); 10.1063/1.5001128

Role of the Fermi level in the formation of electronic band-tails and mid-gap states of hydrogenated amorphous silicon in thin-film solar cells

Journal of Applied Physics 122, 093101 (2017); 10.1063/1.4989425

Vapor-solid-solid grown Ge nanowires at integrated circuit compatible temperature by molecular beam epitaxy Journal of Applied Physics 122, 094304 (2017); 10.1063/1.4990602

Nanopatterned epitaxy of non-polar $\mathrm{Ga}_{1-y} \mathrm{In}_{\mathrm{y}} \mathrm{N}$ layers with caps and voids Journal of Applied Physics 122, 094303 (2017); 10.1063/1.5001126

\section{Instruments for Advanced Science}

W whw. HidenAnalytical com

E info@hiden.co.uk

CLICK TO VIEW our product catalogue

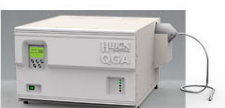

Gas Analysis

dynamic measurement of reaction gas streams , cataysis and thermal analysis

molecular beam studes

dissolved species probes

fermentation, envirormental and ecological stud

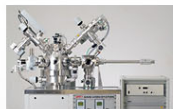

Surface Science

UHVTPD

end point detection in in beam etch elemental limaging -surface mapping

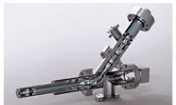

Plasma Diagnostics plasma source characterization. etch and deposition process reaction kinetic studies

analysis of neutral and radical species

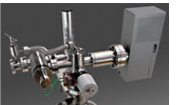

Vacuum Analysis partial pressure measurement and control of process gases reactive sputter process contr vacuum diagnostic 


\title{
Current-mode deep level transient spectroscopy of a semiconductor nanowire field-effect transistor
}

\author{
Ivan Isakov, ${ }^{\text {a) }}$ Marion J. L. Sourribes, and Paul A. Warburton ${ }^{\text {b) }}$ \\ London Centre for Nanotechnology, University College London, 17-19 Gordon Street, London WC1H 0AH, \\ United Kingdom, and Department of Electronic and Electrical Engineering, University College London, \\ London WCIE 7JE, United Kingdom
}

(Received 23 May 2017; accepted 15 August 2017; published online 5 September 2017)

\begin{abstract}
One of the main limiting factors in the carrier mobility in semiconductor nanowires is the presence of deep trap levels. While deep-level transient spectroscopy (DLTS) has proved to be a powerful tool in analysing traps in bulk semiconductors, this technique is ineffective for the characterisation of nanowires due to their very small capacitance. Here, we introduce a new technique for measuring the spectrum of deep traps in nanowires. In current-mode DLTS ("I-DLTS"), the temperaturedependence of the transient current through a nanowire field-effect transistor in response to an applied gate voltage pulse is measured. We demonstrate the applicability of I-DLTS to determine the activation energy and capture cross-sections of several deep defect states in zinc oxide nanowires. In addition to characterising deep defect states, we show that I-DLTS can be used to measure the surface barrier height in semiconductor nanowires. Published by AIP Publishing.
\end{abstract}

[http://dx.doi.org/10.1063/1.5000370]

\section{INTRODUCTION}

Semiconductor nanowires are promising candidates for applications in nanoelectronics. ${ }^{1,2}$ Various nanowire-based devices including field-effect transistors (FETs), ${ }^{3,4}$ sensors, ${ }^{5}$ axial and core-shell heterostructures, ${ }^{6}$ and single-electron transistors ${ }^{7}$ have been reported. The electrical properties of nanowires dramatically depend on defects located both in the nanowire core and on the nanowire surface. ${ }^{8}$ Thus, the understanding of defect behaviour is crucial for controlling nanowire transport properties. The investigation of electrically active deep level defects in bulk semiconductors is usually carried out by means of transient techniques such as deep level transient spectroscopy (DLTS) ${ }^{9}$ and its derivatives (e.g., photoinduced current spectroscopy-PICTS ${ }^{10}$ and current transient spectroscopy ${ }^{11}$ ), thermostimulated current, ${ }^{12}$ electron paramagnetic resonance, and others. Here, the term "deep level" denotes a state that is located deep in the semiconductor band gap (i.e., at energies lower than $E_{C}-k_{B} T$ and higher than $E_{V}+k_{B} T$, with $E_{C}$ and $E_{V}$ being the conduction band minimum and valence band maximum, respectively).

Investigation of the electrically active deep defect states in nanowires by DLTS is a challenging task, mainly due to the difficulties in measuring the very small capacitances of nanowire devices. Only a few papers have therefore been published on nanowire deep levels: DLTS in combination with PICTS was used to study catalyst-related electrical defects in an array of Si nanowires, ${ }^{13}$ DLTS was used to study a single GaN nanowire pn-junction; ${ }^{14}$ and low frequency noise spectroscopy (LFNS) was applied by Motayed et al. to investigate the effects of metal catalysts on Si nanowires. ${ }^{15,16}$

\footnotetext{
${ }^{\text {a)} C u r r e n t ~ a d d r e s s: ~ D e p a r t m e n t ~ o f ~ P h y s i c s, ~ B l a c k e t t ~ L a b o r a t o r y, ~ I m p e r i a l ~}$ College London, London SW7 2AZ, United Kingdom.

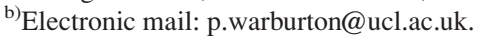

A different approach has been used to study nanoscale devices (such as thin film field-effect transistors), exploiting current-mode DLTS (I-DLTS) as opposed to the conventional capacitance-mode DLTS. ${ }^{17}$ In I-DLTS, the relaxation of the current through the channel in response to a gate voltage pulse is measured. I-DLTS is a non-destructive technique which can be carried out directly on a device in a transistor geometry and does not need any additional fabrication steps. Since nanowire field effect transistors may become constituents of future electronic devices, I-DLTS is a promising technique to study them.

In this paper, we present I-DLTS measurements carried out on individual semiconductor nanowire field effect transistors. Theoretical models for conductivity relaxation driven by states on the nanowire surface are proposed. In addition to the usual characterisation of the deep traps in the semiconductor, an analytical model allows the measurement of the surface band-bending of a $\mathrm{ZnO}$ nanowire. These results corroborate data obtained using surface sensitive techniques such as ultraviolet photoelectron spectroscopy ${ }^{18}$ and surface photovoltage measurements. $^{19}$

\section{A. Current-mode deep level transient spectroscopy in nanowires}

\section{I-DLTS method}

In I-DLTS measurements on nanowire FETs, a fixed drain-source voltage $V_{\mathrm{ds}}$ is applied to the nanowire resulting in a constant current $I_{\mathrm{ds}, 0}$ through the nanowire. The gate voltage is kept at a quiescent value $V_{\mathrm{GQ}}$, and a gate voltage pulse $\Delta V_{\mathrm{G}}$ is applied periodically with period $T_{\mathrm{P}}$ and width $t_{\mathrm{P}}$ [Fig. 1(a)]. In n-type nanowires (like $\mathrm{ZnO}$ ), a positive pulse $\Delta V_{\mathrm{G}}$ populates discrete deep trap states with electrons, which, after the end of the pulse, get emitted from the deep traps with an emission rate $e_{n}$, contributing to the relaxation current $\Delta I(t)=I(t)-I_{\mathrm{ds}, 0}$ through the nanowire [Fig. 1(b)]. 


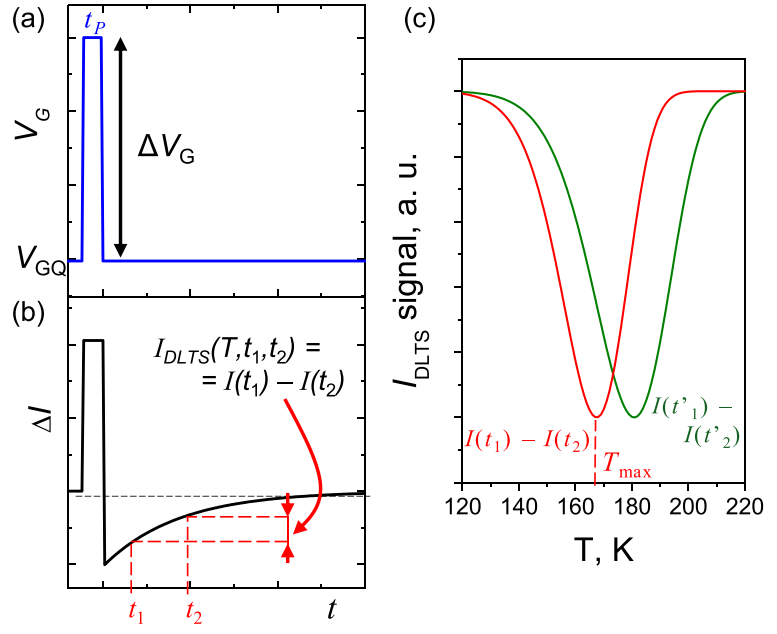

FIG. 1. I-DLTS principle of operation. (a) Time-dependence of the gate voltage; (b) time-dependence of the current through the nanowire; (c) I-DLTS spectra constructed using two different sets of times $\left(t_{1}, t_{2}\right)$ and $\left(t_{1}^{\prime}, t_{2}^{\prime}\right)$.

The I-DLTS signal is constructed by measuring the relaxation current at times $t_{1}$ and $t_{2}$ after the end of the pulse and subtracting them: $I_{\text {DLTS }} \equiv I\left(t_{1}\right)-I\left(t_{2}\right)$.

The rate of carrier emission $e_{n}$ from the deep traps can be expressed as $e_{n} \propto \sigma_{0} \exp \left(-E_{\mathrm{dl}} / k_{\mathrm{B}} T\right)$, where $E_{\mathrm{dl}}$ is the deep trap activation energy, $\sigma_{0}$ the trap cross-section, and $k_{\mathrm{B}}$ Boltzmann's constant. Hence, the emission rate will vary with temperature T. At sufficiently low temperatures, the emission rate will be much lower than $t_{2}^{-1}$, resulting in $I\left(t_{1}\right)-I\left(t_{2}\right)$ being close to zero. Conversely, at high temperatures, the emission rate will be much higher than $t_{1}^{-1}$, also resulting in $I\left(t_{1}\right)$ $-I\left(t_{2}\right)=0$. At some intermediate temperature, $T_{\max }, I_{\text {DLTS }}$ will reach a peak value [Fig. 1(c)]. The value of $T_{\max }$ depends on the choice of $t_{1}$ and $t_{2}$. Assuming an exponential time dependence of the current $\Delta I(t) \propto \exp \left(-e_{n} t\right)$, at temperature $T_{\max }$, there is an unambiguous relationship between the emission rate and the times $t_{1}$ and $t_{2}: e_{n}\left(T_{\max }\right)=\frac{\ln \left(t_{2} / t_{1}\right)}{t_{2}-t_{1}} \cdot{ }^{9}$ By choosing different values of $t_{1}$ and $t_{2}$ and measuring the temperature at which the maximum in $I_{\mathrm{DLTS}}(T)$ occurs, we obtain the dependence of $e_{n}$ on temperature. From this dependence, we can obtain the apparent cross-section of the trap $\sigma_{0}$ and its energy position in the band gap $E_{\mathrm{dl}}$.

It will be shown later that electron emission from the electron traps generally results in a negative current transient signal as shown in Fig. 1(b). This gives rise to an I-DLTS minimum [Fig. 1(c)]. Conversely, hole emission will result in the opposite current transient sign, giving rise to an I-DLTS maximum. Semiconductor nanowires have different types of traps residing in the core of the nanowire, on its surface, and on the semiconductor-dielectric or semiconductor-metal interfaces. These traps will affect the current through the nanowire in different ways. An account of the physical phenomena that govern the dynamics of the carriers due to the deep surface trap states is given in Sec. I A 2.

\section{Current through the nanowire FET}

In general, surface band bending affects the conductivity of a nanowire. For example, $\mathrm{ZnO}$ nanowires have a surface charge depletion layer due to a negative surface charge with the dominant conductivity happening in the core of the nanowire. ${ }^{18,19}$ InAs nanowires, on the other hand, have a surface electron accumulation layer which accounts for the main contribution to the conductivity. ${ }^{20}$

It can be shown (supplementary material) that the current through a back-gated nanowire FET can be expressed as

$$
\begin{aligned}
& I_{\mathrm{ds}}=\frac{\mu_{\mathrm{eff}} C_{\text {oxide }}}{L^{2}}\left[V_{\mathrm{G}}-V_{\mathrm{T}}\right] V_{\mathrm{ds}} \\
& V_{\mathrm{T}}=Q_{\mathrm{ss}} / C_{\text {oxide }}+q \pi R^{2} N_{d} L / C_{\text {oxide }}
\end{aligned}
$$

where $\mu_{\text {eff }}$ is the effective carrier mobility, $V_{\mathrm{G}}$ and $V_{\mathrm{ds}}$ are the gate voltage and drain-source voltage, respectively, $V_{\mathrm{T}}$ is the threshold voltage, $Q_{\mathrm{ss}}$ is the surface trap charge in coulombs, $N_{d}$ is the concentration of ionised shallow donors, $L$ is the distance between contacts, and $R$ is the radius of the nanowire. In general, $Q_{\mathrm{ss}}$ depends on the gate voltage, and we will address this later in this paper. In this paragraph, however, we neglect the gate-voltage dependence of the surface charge. The capacitance $C_{\text {oxide }}$ is calculated based on the model of a metallic wire above a charged plane (supplementary material). Equation (1) coincides well with the usual transistor formula in the linear regime ${ }^{3,21}$ with the additional term $Q_{\mathrm{ss}} / C_{\text {oxide }}$. Because it is usually difficult to estimate the surface state concentration, this extra term can cause ambiguity in the estimation of the ionised donor concentration $N_{d}$. In addition, the analysis is carried out on the system exhibiting axial symmetry. Since this work studies the back-gated nanowires, it should be therefore noted that the current mathematical model is only valid as a first-order approximation.

Figure 2 schematically depicts a standard $\mathrm{ZnO}$ nanowire with a surface depletion region due to negatively charged surface states which are attributed to the absorbed oxygen molecules. Carrier capture onto the surface states depends on the surface barrier height $q V_{\mathrm{B}}=E_{b}$. It can be seen in Figs. 2(a) and 2(b) that the surface barrier for electrons is affected by the gate voltage. The relationship between the gate voltage and the nanowire surface barrier height for a partially depleted nanowire can be expressed as (supplementary material)

$$
V_{\mathrm{B}}\left(V_{\mathrm{G}, \mathrm{eff}}\right)=\frac{q N_{d} W_{d}\left(V_{\mathrm{G}, \mathrm{eff}}\right)\left[2 R-W_{d}\left(V_{\mathrm{G}, \mathrm{eff}}\right)\right] \ln \left(R /\left[R-W_{d}\left(V_{\mathrm{G}, \mathrm{eff}}\right)\right]\right)}{2 \epsilon_{0} \epsilon_{\mathrm{ZnO}}},
$$


(a)

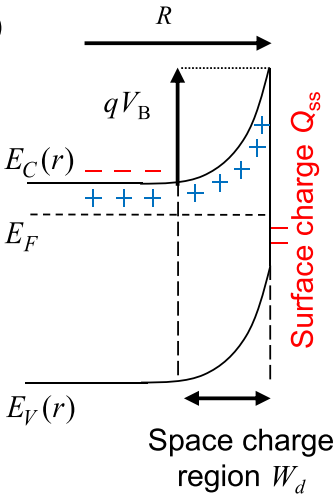

(c)

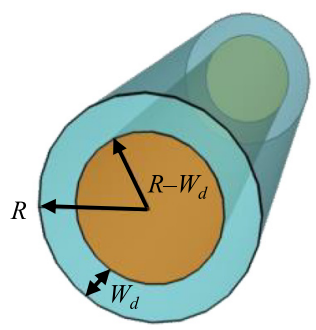

(b)

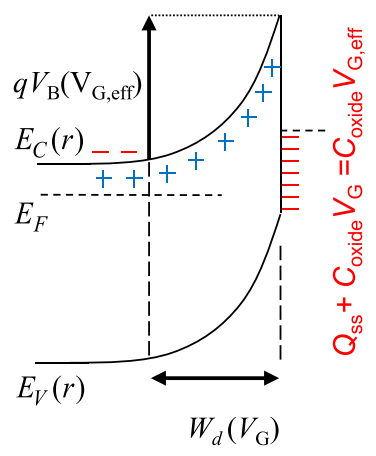

(d)

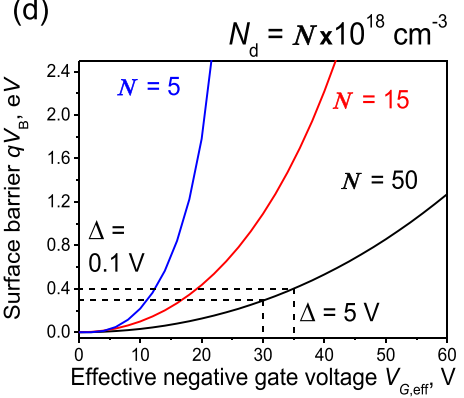

FIG. 2. Model of nanowire band bending for an n-type nanowire with a negative surface charge and surface depletion region: (a) equilibrium condition, $V_{\mathrm{G}}=0$; (b) band diagram of the nanowire under applied negative gate voltage; (c) graphic representation, with the orange region being the conductive core and blue region being the depletion region; and (d) dependence of the surface barrier voltage on the effective negative gate voltage for $\mathrm{ZnO}$ nanowires with different donor concentrations, according to Eq. (2).

$$
W_{d}\left(V_{\mathrm{G}, \mathrm{eff}}\right)=R-\sqrt{R^{2}-\frac{C_{\text {oxide }} V_{\mathrm{G}, \mathrm{eff}}}{q N_{d} \pi L}} .
$$

Here, $V_{\mathrm{G} \text {,eff }}$ is the effective gate voltage $V_{\mathrm{G}, \mathrm{eff}}=Q_{\mathrm{ss}} / C_{\text {oxide }}$ $+V_{\mathrm{G}}$ and $\epsilon_{\mathrm{ZnO}}$ the dielectric constant of $\mathrm{ZnO}$. Figure 2(d) shows the dependence of the barrier height $V_{\mathrm{B}}$ on $V_{\mathrm{G} \text {,eff }}$. In particular, for a typical $\mathrm{ZnO}$ nanowire with a surface barrier of $0.3 \mathrm{eV}$ and with a donor concentration of $N_{d}=5$ $\times 10^{19} \mathrm{~cm}^{-3}$, an increase in the surface barrier by $0.1 \mathrm{eV}$ (i.e., from 0.3 to $0.4 \mathrm{~V}$ ) requires the gate voltage to change by $5 \mathrm{~V}$.

\section{Current transient in nanowires: Emission and capture from the surface trap}

Deep electron traps located in the semiconductor volume (in bulk) will affect the current and capacitance transient behaviour. ${ }^{9}$ The current transients in thin film transistors are affected by both bulk and surface traps. Since nanowires exhibit a very high surface-to-volume ratio, the current transient will predominantly depend on the surface states. Moreover, $\mathrm{ZnO}$ is known to be very surface sensitive, ${ }^{22}$ and therefore, we will consider here only the surface traps.

Let us consider surface electron traps that are uniformly distributed on the surface of the n-type nanowire (Fig. 3). These electron traps have a delta-like density of states with activation energy $E_{\mathrm{ss}}$. At quiescent gate voltage bias $V_{\mathrm{GQ}}$, surface traps below the Fermi energy (with activation energy $\left.E_{\mathrm{ss}, 2}\right)$ are filled with electrons, while those traps above the

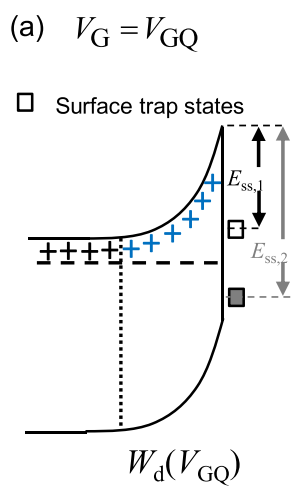
(b) $V_{\mathrm{G}}=V_{\mathrm{GQ}}+\Delta V_{\mathrm{G}}$
Capture mode

(c) $V_{\mathrm{G}}=V_{\mathrm{GQ}}$

Emission mode

FIG. 3. Mechanism of the current transient in nanowires due to surface states: (a) quiescent gate voltage conditions $V_{\mathrm{GQ}}$; (b) positive gate voltage pulse $\Delta V_{\mathrm{G}}$ and electron capture onto surface states; and (c) emission of electrons from the filled traps into the conduction band at the quiescent gate voltage.

Fermi energy (with activation energy $E_{\mathrm{ss}, 1}$ ) are empty [Fig. $3(\mathrm{a})]$. When a positive gate voltage pulse $\Delta V_{\mathrm{G}}$ is applied, the Fermi energy changes its position, with surface traps below the Fermi energy capturing electrons. According to ShockleyRead-Hall statistics, ${ }^{23,24}$ the capture of electrons onto the surface states follows an exponential law with capture rate $c_{n}$ which depends on the surface barrier $q V_{\mathrm{B}}$ [Fig. 3(b)]. The change in time of the number of electrons captured on the surface state $n_{\mathrm{ss}}(t)$ is

$$
\begin{aligned}
n_{\mathrm{ss}}(t) & =n_{\mathrm{ss}, \max }\left(1-\exp \left(-c_{n} t\right)\right) ; \\
c_{n} & =\sigma_{\mathrm{ss}} \gamma T^{2} \exp \left(-q V_{\mathrm{B}} / k_{\mathrm{B}} T\right),
\end{aligned}
$$

where $\sigma_{\mathrm{ss}}$ is the surface state capture cross-section, $n_{\mathrm{ss} \text {, max }}$ the maximum possible number of trapped electrons, and $\gamma$ $=2 \sqrt{3}(2 \pi)^{3 / 2} h^{-3} k^{2} m^{*}$, with $m^{*}$ the effective electron mass. We assume as an approximation that the barrier height $V_{\mathrm{B}}$ is constant in time during the charge capture. It will however depend on the gate voltage as outlined in Sec. I A 2.

At the end of the positive gate voltage pulse, the number of electrons trapped on the surface traps is $n_{\mathrm{ss}, 0}=n_{\mathrm{ss}, \max }$ $\left(1-\exp \left(-c_{n} t_{p}\right)\right)$. When the gate voltage returns to the quiescent bias value, the filled levels start emitting electrons into the conduction band [Fig. 3(c)]. The time-dependence of the number of carriers trapped on the surface levels $n_{\mathrm{ss}}(t)$ is

$$
\begin{aligned}
n_{\mathrm{ss}}(t) & =n_{\mathrm{ss}, 0} \exp \left(-e_{n} t\right) ; \\
e_{n} & =\sigma_{0} \gamma T^{2} \exp \left(-E_{\mathrm{ss}} / k_{\mathrm{B}} T\right),
\end{aligned}
$$

where $E_{\mathrm{ss}}$ is the energy difference between the energy level of the surface trap and the conduction band and $\sigma_{0}$ is an effective minority carrier capture cross section.

The change in population of the surface levels will result in a current transient through the nanowire. Both the surface and bulk traps will effectively change the surface state charge $Q_{\text {ss }}$ in Eq. (1). Surface charge may be represented as a sum of the constant surface charge and the transient surface charge: $Q_{\mathrm{ss}}(t)=Q_{\mathrm{ss}, 0}+q n_{\mathrm{ss}}(t)$. Taking into account the negative charge of electrons for the n-type nanowire, we get the current transient through the nanowire 


$$
\begin{aligned}
\Delta I(t) & =-\frac{V_{\mathrm{ds}} \mu_{\mathrm{eff}}}{L^{2}} \Delta Q_{\mathrm{ss}}(t) \\
\Delta I_{\text {emission }}(t) & =-\frac{\mu q}{L^{2}} V_{\mathrm{ds}} n_{\mathrm{ss}, 0} \exp \left(-e_{n} t\right)=\Delta I_{0} \exp \left(-e_{n} t\right) \\
\Delta I_{0} & =\frac{\mu q}{L^{2}} V_{\mathrm{ds}} n_{\mathrm{ss}, 0} \\
\Delta I_{\text {capture }}(t) & =\frac{\mu q}{L^{2}} V_{\mathrm{ds}} n_{\mathrm{ss}, \max } \exp \left(-c_{n} t\right) .
\end{aligned}
$$

The emission mode and capture mode ${ }^{25}$ I-DLTS may be used to infer trap energies $E_{\mathrm{ss}}$ and the barrier height $V_{\mathrm{B}}$, respectively. Both methods were exploited in this work. Here, we will neglect the temperature dependences of the Fermi level position and the carrier concentration. Therefore, we assume that the values of $n_{\mathrm{ss}, 0}$ and $n_{\mathrm{ss} \text {, max }}$ are independent of temperature for given quiescent gate voltage, gate voltage pulse magnitude, and gate voltage pulse width.

The I-DLTS signal $I_{\text {DLTS }}=I\left(t_{1}\right)-I\left(t_{2}\right)$ is measured at different temperatures and different $t_{1}$ and $t_{2}$ pairs. Every pair will result in an I-DLTS peak: $I_{\text {DLTS, } \max }=I_{\text {DLTS }}\left(T_{\max }\right)$. The magnitude of $I_{\text {DLTS, max }}$ depends on the prefactor $\Delta I_{0}$ in Eq. (6). It can be shown that, if we choose times $t_{1}$ and $t_{2}$ so that $t_{1}$ varies but the ratio $t_{2} / t_{1}$ is fixed, then the magnitude of $I_{\text {DLTS,max }} / I_{d s, 0}(T)$ [where $I_{d s, 0}(T)$ is a quiescent current] corresponding to a specific trap level measured at different times $t_{1}$ will be independent of temperature. In this study, the $t_{2} / t_{1}$ ratio is fixed to 2 and $I_{\text {DLTS,max }}$ will therefore be equal $\Delta I_{0} / 4$ (see supplementary material). The concentration of surface traps $N_{\mathrm{ss}, 0}$, in $\mathrm{cm}^{-2}$, can be calculated from the I-DLTS signal maximum $I_{\text {DLTS, max }}[\mathrm{Eq}$. (6)], and the value of the quiescent current $I_{\mathrm{ds}, 0}$ [Eq. (1)] is given as follows:

$$
\begin{aligned}
N_{\mathrm{ss}, 0} & =\frac{n_{\mathrm{ss}, 0}}{2 \pi R L}=\frac{\Delta I_{0}}{I_{\mathrm{ds}, 0}} \frac{C_{\text {oxide }}\left(V_{\mathrm{G}}-V_{\mathrm{T}}\right)}{q 2 \pi R L} \\
& =\frac{4 \cdot I_{\mathrm{DLTS}, \max }}{I_{\mathrm{ds}, 0}} \frac{C_{\mathrm{ss}}\left(V_{\mathrm{G}}-V_{\mathrm{T}}\right)}{q 2 \pi R L} .
\end{aligned}
$$

The concentration of bulk traps $N_{\text {bulk, } 0}$, in $\mathrm{cm}^{-3}$, can be calculated analogously

$$
N_{\text {bulk }, 0}=\frac{4 \cdot I_{\mathrm{DLTS}, \text { max }}}{I_{\mathrm{ds}, 0}} \frac{C_{\mathrm{ss}}\left(V_{\mathrm{G}}-V_{\mathrm{T}}\right)}{q \pi R^{2} L} .
$$

\section{I-DLTS measurement setup}

The I-DLTS measurement setup is shown in Fig. 4. A $\mathrm{ZnO}$ nanowire field effect transistor with back-gate is used for the measurement. The drain-source voltage through the nanowire is kept constant at $V_{\mathrm{ds}}=0.2 \mathrm{~V}$. A negative quiescent gate voltage bias of $V_{\mathrm{GQ}}=-10 \mathrm{~V}$ keeps a large area of the n-type nanowire cross-section depleted. Gate voltage pulses $\Delta V_{\mathrm{G}}$ of $10 \mathrm{~V}$ amplitude and $100 \mu$ s duration are applied to the gate of the nanowire FET with a repetition rate between 0.1 and $1 \mathrm{kHz}$. The current through the nanowire is probed by the differential pre-amplifier across a reference resistor whose resistance is much smaller than the nanowire resistance. The DC component of the current is filtered out, and the relaxation current is amplified. The signal is supplied to the oscilloscope,

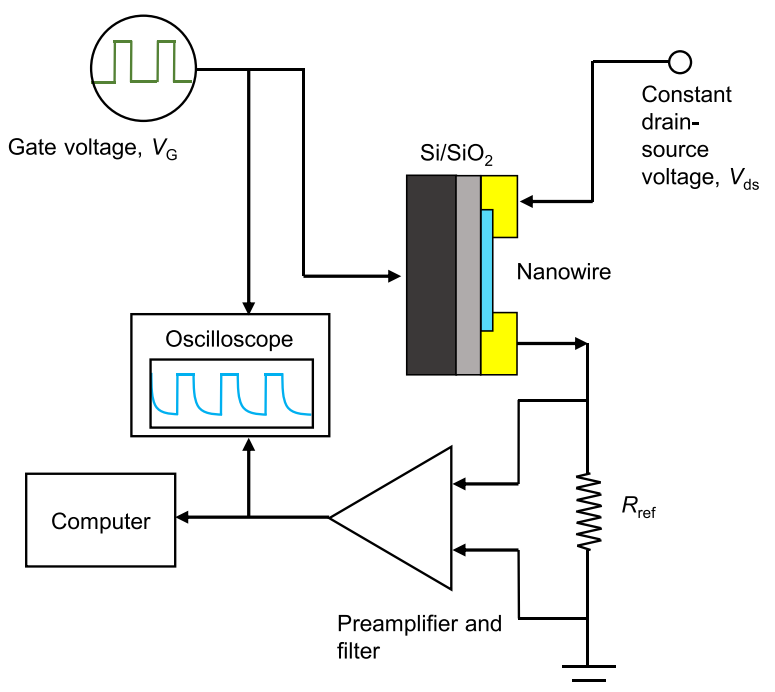

FIG. 4. I-DLTS measurement setup for the nanowire FET.

averaged, and digitised. The time value $t_{1}$ ranges from $50 \mu \mathrm{s}$ to $4 \mathrm{~ms}$.

\section{EXPERIMENTAL DETAILS}

\section{A. ZnO nanowire field effect transistor}

$\mathrm{ZnO}$ nanowires were grown on $\mathrm{Al}_{2} \mathrm{O}_{3}(0001)$ substrates by oxygen plasma assisted molecular beam epitaxy using gold as a catalyst. Details of the growth conditions and nanowire properties are published elsewhere. ${ }^{26}$ Nanowires are $40-100 \mathrm{~nm}$ thick and $1-4 \mu \mathrm{m}$ long. The as-grown sample was ultrasonicated in 2-propanol to remove nanowires from the substrate. A droplet of the nanowire-2-propanol solution was deposited and dried on an oxidised silicon substrate with $120 \mathrm{~nm}$ silicon oxide thickness. Metal contacts to the nanowires were patterned by electron beam lithography. Nanowires were argon ion-beam milled for $30 \mathrm{~s}$, and $\mathrm{Ti} / \mathrm{Au}$ contacts were sputtered onto them [Fig. 5(a)] without breaking vacuum. The Ar ion milling was performed to remove an amorphous surface layer and thereby to decrease the contact resistance. ${ }^{27}$ After fabricating the sample, the substrate was mounted onto the copper block of a chip carrier, which, in turn, was mounted on the end of the dip probe. Liquid helium was used to cool the sample down to $4.2 \mathrm{~K}$.

Room temperature transport characteristics were measured with a Keithley 4200 semiconductor characterization system. The drain-source voltage dependence of the current of a typical $\mathrm{ZnO}$ nanowire is shown in Fig. 5(b), showing Ohmic behaviour. Four-point probe measurements showed a negligible contact resistance. The resistance of the $\mathrm{ZnO}$ nanowires ranged from $100 \mathrm{k} \Omega$ to $1 \mathrm{M} \Omega$. The dependence of the drain source current on the gate voltage is depicted in Fig. 5(c). The on/off ratio was approximately $10^{6}$. The field effect mobility of the nanowire was calculated from the drain-source current using Eq. (1). The nanowire effective mobility at room temperature varied from 10 to $20 \mathrm{~cm}^{2} /(\mathrm{V} \mathrm{s})$ from wire to wire. The effective room-temperature carrier concentration of the nanowires ranged from $10^{18}$ to $10^{19} \mathrm{~cm}^{-3}$. The resistance, mobility, and carrier concentration values are in agreement with data obtained by other researchers. ${ }^{3,18}$ The temperature 


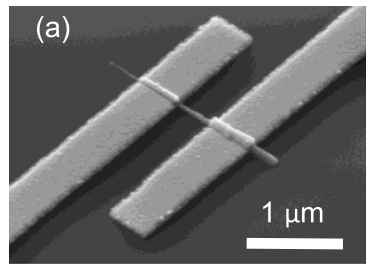

(c)

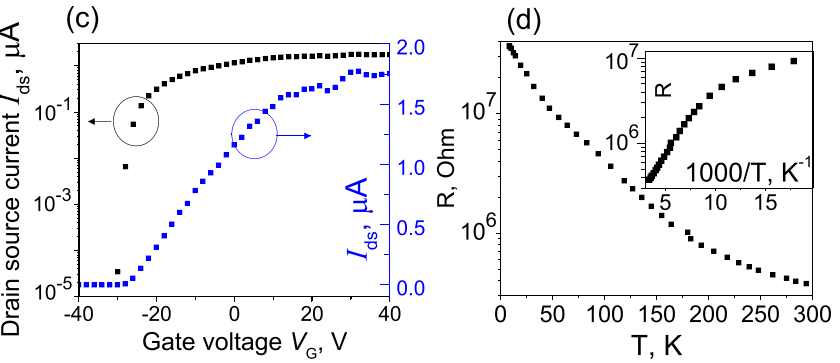

FIG. 5. (a) Scanning electron image of a connected nanowire; (b) room temperature current voltage characteristics of the nanowire at different gate voltages; (c) the gate voltage dependence of drain-source current; and (d) semi-logarithmic plot of the temperature dependence of the nanowire resistance. Inset: resistance on a logarithmic scale as a function of inverse temperature.

dependence of the 2-point probe DC resistance of a single nanowire is shown in Fig. 5(d).

\section{B. I-DLTS: Trap characterisation}

I-DLTS measurements were performed on four $\mathrm{ZnO}$ nanowires. The geometrical and electrical parameters of the studied nanowires are shown in Table I. Current transients at different temperatures for one nanowire are presented in Fig. 6(a). A transient drain current response to a gate voltage pulse similar to the one shown here was previously observed in $\mathrm{ZnO}$ nanowire FETs at room temperature in Ref. 28. Authors attributed the current transients on a time-scale of seconds to adsorption and desorption of oxygen molecules. However, no temperature dependence was carried out. Here, the time-scales are much faster (micro- and milliseconds), and the temperatures are lower. We therefore assume that the current transients in our measurements depend only on the charging and discharging of carrier traps in the nanowire, and the model for the carrier emission given above may be applied for the analysis of these data.

We shall now consider the transients in Fig. 6(a). A short negative relaxation immediately after the end of the gate voltage pulse with a time constant of approximately $50 \mu \mathrm{s}$ is attributed to the equipment response and is similar among

TABLE I. Geometrical and room temperature electrical parameters of the nanowires used in the present study.

\begin{tabular}{lcccc}
\hline \hline Nanowire & $\begin{array}{c}\text { Diameter } \\
(\mathrm{nm})\end{array}$ & $\begin{array}{c}\text { Channel length } \\
(\mathrm{nm})\end{array}$ & $\begin{array}{c}\text { Mobility } \\
\left(\mathrm{cm}^{2} / \mathrm{V} \mathrm{s}\right)\end{array}$ & $\begin{array}{c}\text { Carrier concentration } \\
\left(\mathrm{cm}^{-3}\right)\end{array}$ \\
\hline No. 1 & 60 & 500 & 16.1 & $6.5 \times 10^{18}$ \\
No. 2 & 50 & 500 & 14.7 & $8.5 \times 10^{18}$ \\
No. 3 & 64 & 700 & 15.2 & $3.7 \times 10^{19}$ \\
No. 4 & 49 & 415 & 17 & $1.0 \times 10^{19}$ \\
\hline \hline
\end{tabular}

(a)

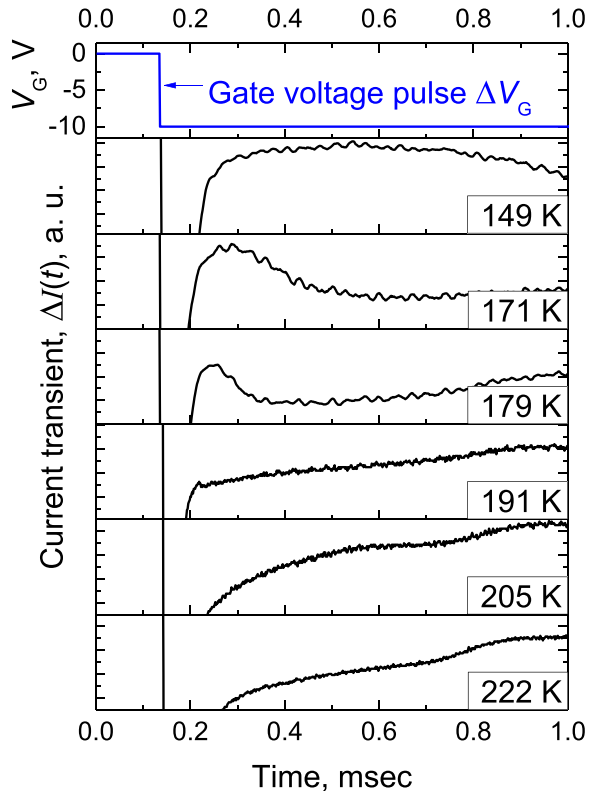

(b)

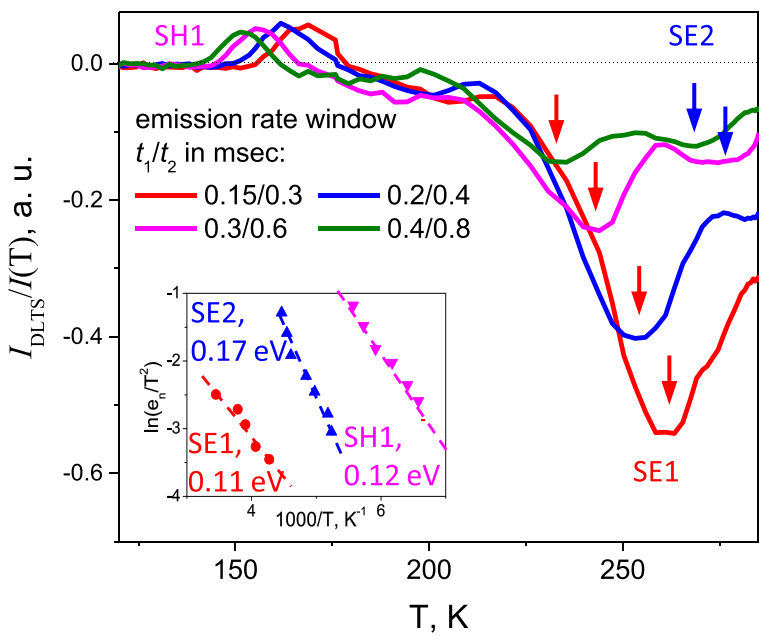

FIG. 6. (a) Current transients of nanowire No.1 at different temperatures for $\Delta V_{\mathrm{G}}=10 \mathrm{~V}$; the negative current peak at $\mathrm{t}<0.1 \mathrm{~ms}$ is due to the equipment response; (b) I-DLTS signal normalized to static current with deep levels indicated. Inset: Arrhenius plots of levels SE1, SE2, and SH1 taken from the I-DLTS graphs in (b).

all the measured samples. The relaxation curves show the temperature-evolution of the current behaviour due to the deep trap. As expected from the exponential behaviour of the emission rate [Eq. (5)], the emission rate increases with temperature. It should be noted that the current transient in Fig. 6(a) becomes both negative and positive: negative at temperatures above $180 \mathrm{~K}$, as expected from the theoretical description of the electron de-trapping [Fig. 1(a)] and positive at temperatures around $150 \mathrm{~K}$, which potentially indicates an unusual behaviour of the charges: either hole de-trapping or electron trapping. The levels responsible for this behaviour are usually called in the literature "hole-like" levels ${ }^{25}$ and will be discussed further.

I-DLTS spectra of $\mathrm{ZnO}$ nanowire No.1 are shown in Fig. 6(b). Peaks in this particular I-DLTS dependence may be attributed to two electron trap levels SE1 and SE2 and one level resembling a hole-like trap SH1 (which is not a real hole trap due to reasons outlined later in this paper). The 
activation energies and apparent capture cross sections of these traps can be determined from the Arrhenius plots [Fig. 6(b), inset]. Parameters of the deep levels measured on four different nanowires are listed in Table II. The nanowires exhibit similar hole-like traps but different electron traps. The normalised amplitude of the traps $I_{\text {DLTS }} / I(T)$ is relatively large (reaching 0.5 for the level SE1 in nanowire No.1), which indicates a high density of surface traps.

The levels observed in this work have several pronounced traits that are not expected from simple bulk deep traps. First, whereas the I-DLTS SH1 magnitude at different rate-windows stays constant at different temperatures, absolute values of the peak magnitudes attributed to the electron traps, SE1, SE2, and SE3, rapidly increase with temperature [Fig. 6(b) shows levels SE1 and SE2]. This rapid increase in I-DLTS peak magnitude was observed in various reports and was attributed to the surface states at the ungated area of a FET. ${ }^{17,29}$ Since the nanowire FETs in the present work are back-gated, the larger part of the nanowire surface is exposed to air, and this affects the I-DLTS signal through the nanowire surface states. This rapid increase in the I-DLTS signal does not allow accurate estimation of the concentration of the SE1, SE2, and SE3 traps.

In addition, we can compare the measured $\mathrm{ZnO}$ nanowire trap signatures with those of the levels studied in bulk $\mathrm{ZnO}$. $\mathrm{ZnO}$ films, bulk crystals, and microwires have been studied by capacitance-mode DLTS with various electron trap levels observed. ${ }^{30-35}$ Although the energies of the levels SE1, SE2, and SE3 coincide with the energies of the levels $\mathrm{E} 1, \mathrm{E} 2$, and E3 from these reports, the trap cross-sections are several orders of magnitude lower in the present work. This observation might indicate the existence of completely different electron traps in the bulk of nanowires or, conversely, a surface origin of levels SE1, SE2, and SE3 due to the potential cross-section reduction at the nanowire surface. This would corroborate our previous assumption of the surface origin of these levels. Level E4, however, coincides in both the energy and cross-section with the level E4 from Refs. 30, 31, and 35, where it is attributed to oxygen vacancies. The E4 trap concentration calculated using Eq. (8) is

TABLE II. Activation energies, apparent cross-section, and concentration of trap levels derived from Arrhenius plots for four $\mathrm{ZnO}$ nanowires. For I-DLTS spectra and Arrhenius plots of all the nanowires (see supplementary material).

\begin{tabular}{lcccc}
\hline \hline Levels Nanowire No. Energy $(\mathrm{eV})$ & Cross-section $\left(\mathrm{cm}^{2}\right)$ & Concentration \\
\hline SE1 & 1,2 & 0.11 & $7 \times 10^{-21}$ & $--^{\mathrm{b}}$ \\
SE2 & 1,2 & $0.15-0.2$ & $4 \times 10^{-19}-2 \times 10^{-18}$ & $--^{\mathrm{b}}$ \\
SE3 & 1 & $0.25-0.3$ & $(1-5) \times 10^{-18}$ & $--^{\mathrm{b}}$ \\
E4 & 4 & 0.45 & $3 \times 10^{-12}$ & $3 \mathrm{D}, 1.3 \times 10^{17} \mathrm{~cm}^{-3}$ \\
SH1 & 1,3 & 0.12 & $8 \times 10^{-19}$ & $2 \mathrm{D}, 2.8 \times 10^{12} \mathrm{~cm}^{-2}$ \\
SH2 & 2 & 0.2 & $3 \times 10^{-18}$ & $2 \mathrm{D}, 4.7 \times 10^{12} \mathrm{~cm}^{-2}$ \\
SH3 & 3 & 0.2 & $2 \times 10^{-19}$ & $2 \mathrm{D}, 1.2 \times 10^{13} \mathrm{~cm}^{-2}$ \\
\hline
\end{tabular}

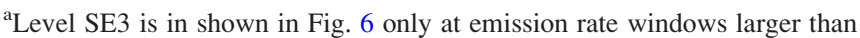
those shown or at higher temperatures (see supplementary material).

${ }^{\mathrm{b}} \mathrm{SE} 1, \mathrm{SE} 2$, and SE3 I-DLTS peak magnitudes increase with temperature, and therefore, the existing model cannot be used for the trap concentration estimation.
$1.3 \times 10^{17} \mathrm{~cm}^{-3}$ which is approximately 300 times less than the carrier concentration of the nanowire.

Levels SH1, SH2, and SH3 show similar activation energies, temperature, and amplitude behaviour and will be examined together. Hole-like levels have been observed in some n-type FETs and are usually ascribed to the surface traps, in particular to hole-traps on the unpassivated surface of the FET (Ref. 25 and references therein). In the case of n-type $\mathrm{ZnO}$ nanowires, it is very unlikely for the levels SH1 to SH3 to be real hole traps; the reasons for this are as follows: For some $\mathrm{ZnO}$ nanowires, the amplitude of the current transient corresponding to the hole-like level is comparable to the static current through the nanowire $\left(I_{\mathrm{I}-\mathrm{DLTS}}\left(T_{\max }\right) / I\left(T_{\max }\right)\right.$ $\approx 0.1-0.5$ ); assuming the hole-like level being real hole traps would indicate the concentration of holes comparable to that of electrons. On the contrary, no inversion current was observed in the FET transfer characteristics at large negative gate biases down to $-60 \mathrm{~V}$, indicating a negligible concentration of holes. Therefore, the levels SH1 to SH3 cannot be real hole traps. Assuming a surface origin of the SH1-SH3 traps, the trap concentration can be estimated from Eq. (7) to be between 2.8 and $12 \times 10^{12} \mathrm{~cm}^{-2}$, equivalent to one surface trap per 3 to $6 \mathrm{~nm}$ of length. Further study is needed to fully understand the origin of the levels SH1 to SH3.

Another phenomenon that can in principle account for the trapping and detrapping of electrons is tunnelling through the surface barrier. The depletion region calculated from Eq. (3) ranges from 3 to $10 \mathrm{~nm}$ which may be low enough for tunnelling effects to take place. It should be noted, however, that the tunnelling probability does not include any direct temperature dependence while being solely a function of the barrier height and the depletion region width. Although the temperature dependence may be included in the barrier height or depletion region width (all these values depend on the carrier concentration), we do not expect it to be exponential. Therefore, the fast temperature dependence of the emission or capture rates observed in I-DLTS curves cannot be explained by tunnelling effects.

In conclusion, all the levels observed in $\mathrm{ZnO}$ nanowire transistor I-DLTS, except the E4 level, appear to be surface state related. This corroborates our initial assumption about $\mathrm{ZnO}$ nanowires being mostly affected by the surface states. Because we cannot unambiguously determine the exact origin of the surface levels, we have to restrict our conclusions to the phenomenological description of their recharging characteristics. The only bulk level E4 is tentatively ascribed to the oxygen vacancies in the nanowire core. Independent of the traps' origin, we can extrapolate the emission rate and characteristic decay time to room temperature using Eq. (5). The time decay of electron detrapping ranges from 0.1 to $200 \mu \mathrm{s}$ (SE1: $120 \mu \mathrm{s}, \mathrm{SE} 2: 10 \mu \mathrm{s} ; \mathrm{SE} 3: 200 \mu \mathrm{s}, \mathrm{E} 4: 0.1 \mu \mathrm{s}$, and SH1-SH3: 5-50 $\mu \mathrm{s})$. These traps will therefore have different effects on the frequency response of future nanowiretransistors in electronic devices.

It should be noted that even though the nanowire bulk properties such as carrier concentration and mobility are similar among the four studied nanowires (Table I), the trap spectra vary significantly. Similar variability in nanowire electrical properties was observed previously in $\mathrm{ZnO}$ nanowires ${ }^{36}$ and 
was attributed to the variation in the density and occupancy of surface defects. The authors claim that this variability is intrinsic to $\mathrm{ZnO}$ nanowires. I-DLTS data presented in the current paper supports the predisposition of $\mathrm{ZnO}$ nanowires to electrical property variability.

\section{I-DLTS: Surface barrier height measurement}

As it was outlined in Sec. I A 3, capture mode I-DLTS may provide information on the nanowire surface barrier height. Any surface state that can trap electrons at positive gate voltages and de-trap electrons at negative gate voltages can be used for this purpose [Eq. (4)]. The surface barrier height depends on the effective gate voltage [Fig. 3(b.2), Eq. (2)], and therefore, temperature of the capture-mode I-DLTS peak will depend on the quiescent gate voltage value as well.

Capture-mode I-DLTS was carried out on nanowire sample No. 4 (Fig. 7). Figure 7(a) shows capture-mode I-DLTS spectra at two different quiescent voltages with the most prominent peaks at $220 \mathrm{~K}$ and $230 \mathrm{~K}$. We assume that these two peaks correspond to the process of carrier capture over the surface barrier by the same surface trap level which we denote by $\mathrm{C} 1$. The surface barrier heights obtained from the I-DLTS measurements are $0.3 \mathrm{eV}$ and $0.4 \mathrm{eV}$ (for $V_{\mathrm{GQ}}=10 \mathrm{~V}$ and $V_{\mathrm{GQ}}=0 \mathrm{~V}$ respectively, see supplementary material). These values of the surface band bending in the $\mathrm{ZnO}$ nanowire

(a)

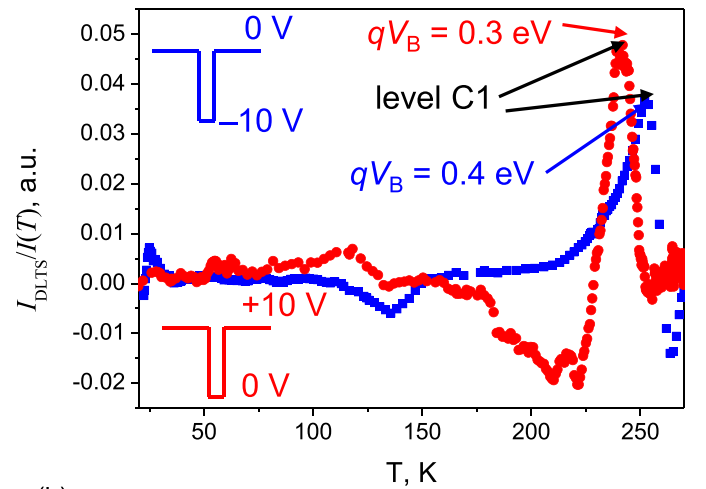

(b)

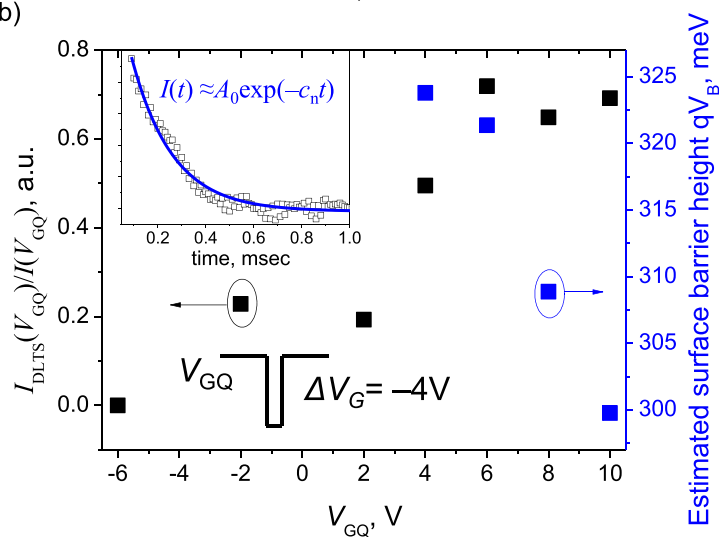

FIG. 7. (a) Capture-mode I-DLTS measurement on a $\mathrm{ZnO}$ nanowire with different quiescent gate voltages: $V_{\mathrm{GQ}}=+10 \mathrm{~V}$ (red) and $V_{\mathrm{GQ}}=0 \mathrm{~V}$ (blue); the emission rate constant $t_{1} / t_{2}$ was $0.4 / 0.8 \mathrm{~ms}$. Hole-like peaks are observed at $220-240 \mathrm{~K}$. (b) Quiescent voltage dependence of the capture-mode IDLTS signal and capture activation energy; the gate voltage pulse is $-4 \mathrm{~V}$. Inset: current transient at $V_{\mathrm{GQ}}=10 \mathrm{~V}, \Delta V_{\mathrm{G}}=-4 \mathrm{~V}$; the blue line represents the exponential fit to the experimental data. are similar to those obtained by Soudi et al..$^{19}(0.3 \mathrm{eV})$ and of the same order as data obtained by Chen et al. ${ }^{18}(0.74 \mathrm{eV})$. The differences might arise due to different growth methods used.

According to the model in Fig. 3(b.2), the level C1 exhibits a varying capture activation energy (and consequently the surface barrier height) which depends on the quiescent voltage. The surface barrier $q V_{\mathrm{B}}$ decreases by $0.1 \mathrm{eV}$ (from $0.4 \mathrm{eV}$ to $0.3 \mathrm{eV}$ ) when the quiescent voltage increases from 0 to $+10 \mathrm{~V}$ [Fig. 7(a)]. This value is on the same order of magnitude with that obtained from the model described in Sec. IA 2 [Fig. 2(d)]. The capture cross-section $\sigma_{\text {ss }}$ stays approximately the same and is equal to $5 \times 10^{-18} \mathrm{~cm}^{2}$.

Figure $7(\mathrm{~b})$ shows a more detailed quiescent voltage dependence of the I-DLTS signal magnitude, with a fixed gate voltage pulse of $\Delta \mathrm{V}_{\mathrm{G}}=-4 \mathrm{~V}$. The peak increases with quiescent voltage. Since the capture follows an exponential time-dependence [Fig. 7(b), inset], the capture time-constant can be inferred from the fit. It changes from 0.5 to $0.17 \mathrm{~ms}$ when the quiescent voltage increases from +4 to $+10 \mathrm{~V}$. The capture activation energy (or barrier energy $E_{b}$ ) is calculated using Eq. (4) for $\mathrm{V}_{\mathrm{GQ}}>4 \mathrm{~V}$ and plotted in Fig. 7(b) (blue squares). The barrier energy monotonically decreases from 325 to $300 \mathrm{meV}$ as expected from the depletion region model.

We can estimate the surface charge concentration at $V_{G}=0$ from the barrier height [Eqs. (2) and (3)]. Using a carrier concentration value of $10^{19} \mathrm{~cm}^{-3}$ (Table I), the surface charge density at zero gate bias is $3.5 \times 10^{12} \mathrm{~cm}^{-2}$.

\section{CONCLUSIONS AND PROSPECTS}

In conclusion, we have proposed and successfully implemented the I-DLTS method with gate-voltage pulsing to probe deep electronic states in individual $\mathrm{ZnO}$ nanowires. This method offers an effective tool to study energetics of electron trapping in nanowire surface states. As an illustration of the nanowire I-DLTS method, $\mathrm{ZnO}$ nanowire field-effect transistors were studied. A variety of deep levels were observed, with both electron-like and hole-like characters. A comparison with the literature showed that levels SE1, SE2, and SE3 are most likely surface trap levels, whereas E4 is a bulk nanowire level related to oxygen vacancies. Different types of I-DLTS measurement setups (emission-mode, capture-mode, and quiescent voltage dependence) were applied to the nanowire FETs. The surface barrier was estimated from the capturemode I-DLTS to be $0.3-0.4 \mathrm{eV}$. The hole-like trap levels were discovered to affect all the nanowire FET transients.

I-DLTS in conjunction with sample post-treatment and other characterisation methods can improve understanding of the effect of gas molecule adsorption on the surface electronic structure, passivation effects in core-shell nanowires, mechanisms of water splitting on the surface of nanostructures, and incorporation of dopants in the nanowires. Numerical models may be refined to include tunnelling effects, chemical structure, and band gap tail traps. Development of a more sophisticated analysis of the quiescent gate-voltage sweeping technique on wrap-gate FETs may give information on the spatial location of defect states in nanowires with a non-uniform composition (such as axial and core-shell nanowires). Information inferred 
using this method can help research into better chemical nanowire-based sensors, photodiodes, memory devices, and stable transistors. Although further development is still necessary, I-DLTS has the potential to become a versatile method for nanowire trap state analysis.

\section{SUPPLEMENTARY MATERIAL}

See supplementary material for the derivation of the equation for the current through the nanowire FET, I-DLTS peak magnitude and I-DLTS spectra, and Arrhenius plots of all the studied nanowires.

\section{ACKNOWLEDGMENTS}

This work was funded by EPSRC grant reference EP/ H005544/1. I would like to thank Dr. Ed Romans and Professor Oleg Vyvenko for fruitful discussions on the DLTS measurement setup and analysis of the data.

${ }^{1}$ J. Cui, “Zinc oxide nanowires,” Mater. Charact. 64, 43-52 (2012).

${ }^{2}$ Y. Li, F. Qian, J. Xiang, and C. M. Lieber, "Nanowire electronic and optoelectronic devices," Mater. Today 9(10), 18-27 (2006).

${ }^{3}$ J. Goldberger, D. J. Sirbuly, M. Law, and P. Yang, "ZnO nanowire transistors," J. Phys. Chem. B 109(1), 9-14 (2005).

${ }^{4}$ S. Dhara, S. Sengupta, H. S. Solanki, A. Maurya, R. Arvind Pavan, M. R. Gokhale, A. Bhattacharya, and M. M. Deshmukh, "Facile fabrication of lateral nanowire wrap-gate devices with improved performance," Appl. Phys. Lett. 99(17), 173101 (2011).

${ }^{5}$ Q. Wan, Q. Li, Y. Chen, T. Wang, X. He, J. Li, and C. Lin, "Fabrication and ethanol sensing characteristics of $\mathrm{ZnO}$ nanowire gas sensors," Appl. Phys. Lett. 84, 3654-3656 (2004).

${ }^{6}$ M. T. Björk, B. J. Ohlsson, T. Sass, A. I. Persson, C. Thelander, M. H. Magnusson, K. Deppert, L. R. Wallenberg, and L. Samuelson, "One-dimensional steeplechase for electrons realized," Nano Lett. 2(2), 87-89 (2002).

${ }^{7}$ C. Thelander, T. Mårtensson, M. T. Björk, B. J. Ohlsson, M. W. Larsson, L. R. Wallenberg, and L. Samuelson, "Single-electron transistors in heterostructure nanowires," Appl. Phys. Lett. 83(10), 2052-2054 (2003).

${ }^{8}$ M. J. L. Sourribes, I. Isakov, M. Panfilova, H. Liu, and P. A. Warburton, "Mobility enhancement by Sb-mediated minimisation of stacking fault density in InAs nanowires grown on silicon," Nano Lett. 14(3), 1643-1650 (2014).

${ }^{9}$ D. V. Lang, "Deep-level transient spectroscopy: A new method to characterize traps in semiconductors," J. Appl. Phys. 45(7), 3023-3032 (1974).

${ }^{10}$ G. M. Martin and D. Bois, Proc. Electrochem. Soc. 78, 32 (1978).

${ }^{11}$ J. A. Borsuk and R. Swanson, "Current transient spectroscopy: A highsensitivity DLTS system," IEEE Trans. Electron Devices 27, 2217-2225 (1980).

${ }^{12}$ J. G. Simmons and G. W. Taylor, "High-field isothermal currents and thermally stimulated currents in insulators having discrete trapping levels," Phys. Rev. B 5, 1619-1629 (1972).

${ }^{13}$ K. Sato, A. Castaldini, N. Fukata, and A. Cavallini, "Electronic level scheme in boron- and phosphorus-doped silicon nanowires," Nano Lett. 12(6), 3012-3017 (2012).

${ }^{14}$ Y. S. Park, C. M. Park, C. J. Park, H. Y. Cho, S. J. Lee, T. W. Kang, S. H. Lee, J.-E. Oh, K.-H. Yoo, and M.-S. Son, "Electron trap level in a GaN nanorod p-n junction grown by molecular-beam epitaxy," Appl. Phys. Lett. 88(19), 192104 (2006).

${ }^{15}$ A. Motayed, S. Krylyuk, and A. V. Davydov, "Characterization of deeplevels in silicon nanowires by low-frequency noise spectroscopy," Appl. Phys. Lett. 99, 113107 (2011).
${ }^{16}$ D. Sharma, A. Motayed, S. Krylyuk, Q. Li, and A. Davydov, "Detection of deep-levels in doped silicon nanowires using lowfrequency noise spectroscopy," IEEE Trans. Electron Devices 60, 4206-4212 (2013).

${ }^{17} \mathrm{Z}$. Li, "Systematic modelling and comparisons of capacitance and currentbased microscopic defect analysis techniques for measurements of highresistivity silicon detectors after irradiation," Nucl. Instrum. Methods Phys. Res., Sect. A 403(2-3), 399-416 (1998).

${ }^{18}$ C.-Y. Chen, J. R. D. Retamal, I.-W. Wu, D.-H. Lien, M.-W. Chen, Y. Ding, Y.-L. Chueh, C.-I. Wu, and J.-H. He, "Probing surface band bending of surface-engineered metal oxide nanowires," ACS Nano 6(11), 9366-9372 (2012).

${ }^{19}$ A. Soudi, C.-H. Hsu, and Y. Gu, "Diameter-dependent surface photovoltage and surface state density in single semiconductor nanowires," Nano Lett. 12(10), 5111-5116 (2012).

${ }^{20}$ C. Blömers, T. Grap, M. I. Lepsa, J. Moers, S. Trellenkamp, D. Grützmacher, H. Lüth, and T. Schäpers, "Hall effect measurements on InAs nanowires," Appl. Phys. Lett. 101(15), 152106 (2012).

${ }^{21}$ S. M. Sze, Physics of Semiconductor Devices (Wiley Intescience, New York, London, Sydney, Toronto, 1969).

${ }^{22}$ M.-W. Ahn, K.-S. Park, J.-H. Heo, J.-G. Park, D.-W. Kim, K. J. Choi, J.H. Lee, and S.-H. Hong, "Gas sensing properties of defect-controlled ZnO-nanowire gas sensor," Appl. Phys. Lett. 93(26), 263103 (2009).

${ }^{23} \mathrm{~W}$. Shockley and W. T. Read, "Statistics of the recombinations of holes and electrons," Phys. Rev. 87, 835-842 (1952).

${ }^{24}$ R. N. Hall, "Electron-hole recombination in germanium," Phys. Rev. 87, 387-387 (1952)

${ }^{25}$ A. Cavallini, G. Verzellesi, A. F. Basile, C. Canali, A. Castaldini, and E. Zanoni, "Origin of hole-like peaks in current deep level transient spectroscopy of n-channel AlGaAs/GaAs heterostructure field-effect transistors," J. Appl. Phys. 94(8), 5297-5301 (2003).

${ }^{26}$ I. Isakov, M. Panfilova, M. J. L. Sourribes, and P. A. Warburton, "Growth of $\mathrm{ZnO}$ and $\mathrm{ZnMgO}$ nanowires by Au-catalysed molecular-beam epitaxy," Phys. Status Solidi C 10(10), 1308-1313 (2013).

${ }^{27}$ M. J. L. Sourribes, I. Isakov, M. Panfilova, and P. A. Warburton, "Minimization of the contact resistance between InAs nanowires and metallic contacts," Nanotechnology 24(4), 045703 (2013).

${ }^{28}$ J. Maeng, W. Park, M. Choe, G. Jo, Y. H. Kahng, and T. Lee, "Transient drain current characteristics of $\mathrm{ZnO}$ nanowire field effect transistors," Appl. Phys. Lett. 95(12), 123101 (2009).

${ }^{29}$ J. H. Zhao, "Modeling the effects of surface states on DLTS spectra of GaAs MESFETs," IEEE Trans. Electron Devices 37(5), 1235-1244 (1990).

${ }^{30}$ F. D. Auret, S. A. Goodman, M. J. Legodi, W. E. Meyer, and D. C. Look, "Electrical characterization of vapor-phase-grown single-crystal $\mathrm{ZnO}$," Appl. Phys. Lett. 80(8), 1340-1342 (2002).

${ }^{31}$ V. Quemener, L. Vines, E. V. Monakhov, and B. G. Svensson, "Evolution of deep electronic states in $\mathrm{ZnO}$ during heat treatment in oxygen- and zinc-rich ambients," Appl. Phys. Lett. 100(11), 112108 (2012).

${ }^{32}$ F. Schmidt, S. Müller, H. von Wenckstern, C. P. Dietrich, R. Heinhold, H.-S. Kim, M. W. Allen, and M. Grundmann, "Comparative study of deep defects in $\mathrm{ZnO}$ microwires, thin films and bulk single crystals," Appl. Phys. Lett. 103(6), 062102 (2013).

${ }^{33}$ V. Quemener, L. Vines, E. V. Monakhov, and B. G. Svensson, "Electrical characterization of hydrothermally grown $\mathrm{ZnO}$ annealed in different atmospheres," Int. J. Appl. Ceram. Technol. 8(5), 1017-1022 (2011).

${ }^{34}$ A. Hupfer, C. Bhoodoo, L. Vines, and B. G. Svensson, "The E3 center in zinc oxide: Evidence for involvement of hydrogen,” Appl. Phys. Lett. 104(9), 092111 (2014).

${ }^{35}$ D. Seghier and H. P. Gislason, "On intrinsic defects in Co-doped $\mathrm{ZnO}$ thin films," Phys. Scr. 2010(T141), 014007.

${ }^{36}$ A. M. Lord, T. G. Maffeis, A. S. Walton, D. M. Kepaptsoglou, Q. M. Ramasse, M. B. Ward, J. Köble, and S. P. Wilks, "Factors that determine and limit the resistivity of high-quality individual $\mathrm{ZnO}$ nanowires," Nanotechnology 24(43), 435706 (2013). 